\title{
COGNITIVE, PERCEPTUAL AND MOTOR DECLINE AS PREDICTORS OF RISKY STREET-CROSSING DECISIONS IN OLDER PEDESTRIANS
}

\author{
Sabine Langevin ${ }^{1}$, Aurèlie Dommes ${ }^{1}$, Viola Cavallo ${ }^{1}$, Jennifer Oxley ${ }^{2}$, \& Fabrice Vienne ${ }^{3}$ \\ ${ }^{1}$ LPC, French Institute of Science and Technology for Transport, \\ Development and Networks (IFSTTAR - INRETS), Versailles, France \\ ${ }^{2}$ MUARC, Monash University Sunway Campus, Malaysia \\ ${ }^{3}$ LEPSIS, French Institute of Science and Technology for Transport, \\ Development and Networks (IFSTTAR - INRETS), Paris, France \\ Email: sabine.langevin@ifsttar.fr
}

\begin{abstract}
Summary: Older pedestrians are well known to be over-involved in road crashes compared to younger pedestrians. This study investigates the extent to which risky street-crossing decisions in older pedestrians can be explained by agerelated declines of cognitive, perceptual and physical abilities. Three age groups of participants (young, young-old, old-old) were evaluated in a street-crossing task and performed a series of functional tests. The results showed that agerelated slowing in walking speed as well as a decline in cognitive flexibility and in visual acuity play a substantial role in risky decisions by the elderly. The implications of these findings, particularly in the development of a mixed physical-cognitive training to enhance the older pedestrians' road crossing decisions in complex environments, are discussed.
\end{abstract}

\section{INTRODUCTION}

For over a decade a number of studies have investigated age differences in street-crossing behaviors that may account for higher crash risks in older pedestrians. Previous research has pointed out greater difficulty in selecting safe gaps to cross and adopting sufficient safety margins amongst older adults, especially when vehicles are approaching at a high speed (Lobjois and Cavallo, 2007, 2009; Oxley et al, 2005;) and/or in two-way traffic situations (see e.g., Holland and Hill, 2010; Oxley et al., 1997).

Risky street-crossing decisions in older pedestrians have often been explained by age-related declines in physical, cognitive and/or perceptual abilities (Oxley et al., 2005; Lobjois and Cavallo, 2007; 2009; Holland and Hill, 2010). Similar findings are noted for older drivers, however, in contrast to driving performance research (Anstey et al., 2005), empirical evidence for road-crossing behaviour is still scarce. A greater understanding of the role of functional declines on crossing decisions is required, particularly in complex environments such as twoway roads where collisions are most likely to occur. To cross a two-way road safely, pedestrians must pay attention to approaching vehicles from both directions. They have to detect cars, determine their direction and velocity and estimate if they have enough time to cross the road completely before the approaching cars reach their crossing line. Pedestrians must thus base their decisions on both sources of traffic information, select the most appropriate ones and inhibit the non relevant information, as well as compare available vehicle arrival times in both lanes to their own time needed to cross. 
Aging is clearly established to be associated with a decline in functional abilities. More specifically, visual perception is affected as shown by deteriorations of the visual field of view (Johnson and Keltner, 1983), contrast sensitivity (Rubin et al., 1997) and motion perception (Hills, 1975). Older people tend to be less able to perceive visual details, to detect motion or position in depth or to cope with adverse light conditions. They also show deficits in processing visual and spatial information to establish complex relationships among objects in their environment. Given that crossing the street is a highly visual-dependent task (Shinar and Scheiber, 1991), the decline in visual perception abilities among older adults, may be an important factor in explaining the higher likelihood of unsafe decisions by seniors compared with younger adults, especially in two-way traffic situations where visual information has to be perceived in both directions.

A decline of several cognitive functions is also associated with aging: aging is characterized by a slowing down of processing speed. Examination of performance on reaction time tasks show that older people respond more slowly than their younger counterparts, with this slowing down being more marked in complex tasks (Salthouse, 1996). Extensive data have also demonstrated a robust decline in executive and attentional functions among elderly people: planning, control of interference, as well as flexibility to switch attentional focus on specific features have been found to be particularly vulnerable to increasing age (Crawford et al., 2000), while executive functions seem highly relevant for competent street-crossing decisions in complex traffic situations such as two-way roads.

Elderly people also suffer from general physical weakening: they lose agility, endurance and musculo-skeletal wasting. Balance control mechanisms and postural reflexes are affected and result in impaired mobility, thus increasing the risk of falls. Not surprisingly, most studies addressing the role of physical changes on pedestrian safety report a significant slowing of walking speed (Oxley et al., 1997; Lobjois and Cavallo, 2007) and smaller steps in older pedestrians (Ketchman and Stelmatch, 2001). As recently suggested by the study of Holland and Hill (2010), physical frailty and reduced mobility associated with aging are some of the factors together with perceptual and cognitive declines that may impair older pedestrians' ability to cross a two-way road safely.

Research conducted in the field of aging suggests that the perceptual, cognitive and physical vulnerability of the older adults has implications for the safety of the decisions they take when crossing a two-way road. However, investigations of the role of these declines on the ability to make appropriate and safe crossing decisions in complex road environments are scarce and lack a multi-dimensional framework. Hence, the aim of this study was to investigate the impact of age-related declines in perceptual, cognitive and physical abilities on the propensity of older pedestrians to experience gap selection difficulties in two-way traffic situations.

\section{METHOD}

Three groups of adults took part in a street-crossing estimation task and underwent a set of cognitive, perceptive and physical tests. 


\section{Participants}

A total of 59 participants took part in the experiment: 20 Young adults ranging in age between 20-35 years (mean 28.9; SD 4.21); 19 Young-Old adults ranging in age between 60-67 years (mean 62.50; SD 2.41) and 20 Old-Old adults ranging between 68-84 years of age (mean 76.55; SD 4.44). The participants in each age group were equally distributed with respect to gender.

\section{Experimental setup and materials}

The street-crossing simulation device was set up to reproduce the demands of a street-crossing task with traffic approaching from one or two ways. The visual scenes were retro-projected on 6 screens each sized $255 \mathrm{~cm}$ (height) $x 188 \mathrm{~cm}$ (width) positioned in a semicircle around the pedestrian, in such a way that $\mathrm{s} /$ he could turn his/her head to the left or right to watch the traffic coming from either of the two directions. Road markers indicated the participant's position on the ground, i.e. in the centre of the immersive simulator and corresponding to the participant's virtual position on the sidewalk. The traffic consisted of flows of motorcycles and cars (from 5 to 10). Vehicle speed (40 and $60 \mathrm{~km} / \mathrm{h}$ ) and time gap between two target cars of the traffic flow (1 to $7 \mathrm{~s}$, in 1-s increments) were systematically varied. Two levels of traffic complexity were presented (one-way and two-way traffic). Direction of the traffic respected the French traffic rules.

In addition, all participants underwent a series of perceptual, cognitive and motor tests. Visualperceptual abilities were assessed using a visual acuity test (Ergovision) and a motion discrimination task (for a description, see Dommes \& Cavallo, in press). Several cognitive functions were assessed: flexibility (Trail Making Test Part B [TMTB] and a subtest of the Test of Attentional Performance [TAP], Zimmerman and Fimm, 2009); inhibition (Go/noGo subtest of the TAP, and the inhibitory subtest of TAP); attention (divided attention subtest of the TAP and UFOV test, parts 2 and 3); and processing speed (CODE subtest of the Wechsler's scale and the Trail Making Test Part A [TMTA], Reitan and Wolfson, 1985). Finally, physical abilities were evaluated using a measure of the walking speed. In a corridor next to the simulator room, each participant stood on a marker and was asked to walk 6 meters at normal walking pace. The average of three measures was used in subsequent analyses.

\section{Procedure}

The experiment was conducted during two sessions of about $1 \mathrm{hr} 30 \mathrm{~min}$ each. Participants performed the street-crossing task in the first session. They were positioned in front of the streetcrossing simulator, and had to choose available gaps in the approaching traffic where they would have 'crossed' the street, by walking but without running or stopping in the middle of the two lanes. To indicate whether the participant thought $\mathrm{s} /$ he could cross, s/he pressed the responsebutton, otherwise s/he waited the next trial. Each participant performed a total of 162 trials, divided up into 4 blocks, with a break between blocks. The order of trials within blocks was randomized and the order of blocks was counterbalanced. Participants then undertook the series of perceptual, cognitive and physical tests in the second session. 


\section{RESULTS}

Street-crossing decisions. All trials were scored according to whether the participant indicated whether or not they would have crossed. The analyses for this paper were focused on the most dangerous type of street-crossing decisions: those that may have resulted in a potential collision. From the positions of the vehicles on the virtual road, the mean reaction time and walking speed of the participant, a potential collision was counted when he/she would have been hit by the virtual approaching car. These data were then expressed as a proportion of all crossing decisions. A one-way ANOVA revealed a significant effect of Age on the percentage of collisions $\left[\mathrm{F}(2,56)=16.38, \mathrm{p}<.0001, \eta^{2}=.37\right]$. The Newman-Keuls post-hoc analysis showed that the old-old group (mean 35.46; SD 24.76) made significantly more decisions that would have resulted in a collision compared with both the young group (mean 7.70; SD 8.92) and the young-old group (mean 16.62; SD 20.34) $(\mathrm{p}<.001)$.

Functional performance. Walking speed was expressed in meters per second ( $\mathrm{m} / \mathrm{s})$. Regarding perceptual tests, a visual acuity score was calculated, and a threshold was computed to determine the duration (in ms) at which the participant could correctly perform the motion perception task $75 \%$ of the time. Regarding each cognitive test, the score reported was the number of omissions or errors, except for the two tests assessing processing speed: correct responses were considered on the CODE test and response time on the TMTA. As two measures for each of the four cognitive abilities were collected (i.e. flexibility, inhibition, attention and processing speed), four composite z-scores were respectively calculated.

A one-way $\mathrm{Age}_{3}$ ANOVA was performed on each of these indicators. The results revealed that all indicators decreased significantly with aging $(\mathrm{p}<.05)$, with a specific and more marked decrease of functional performance in the old-old group compared to young-old or young participants.

The role of functional performance in the safety of street-crossing decisions. To examine the relationships between cognitive, perceptual and physical abilities and collisions, we computed correlations and regressions analyses. The matrix of correlations is presented below in Table 1.

Table 1. Correlation matrix between age, collisions, perceptual, cognitive and physical performances

\begin{tabular}{|c|c|c|c|c|c|c|c|c|c|}
\hline & Age & 1 & 2 & 3 & 4 & 5 & 6 & 7 & 8 \\
\hline \multicolumn{10}{|l|}{ Perceptual measure } \\
\hline 1 Visual acuity & $-.37 *$ & - & & & & & & & \\
\hline 2 Motion discrimination & $.29 *$ & & - & & & & & & \\
\hline \multicolumn{10}{|l|}{ Cognitive measures } \\
\hline 3 Inhibition & $.77 *$ & .07 & .17 & - & & & & & \\
\hline 4 Flexibility & $.42 *$ & $-.26^{*}$ & .24 & $.40 *$ & - & & & & \\
\hline 5 Attention & $.68^{*}$ & $-.33 *$ & $.38^{*}$ & $.65^{*}$ & $.55^{*}$ & - & & & \\
\hline 6 Processing speed & $-.35^{*}$ & .15 & $-.36^{*}$ & $-.34 *$ & $-.38^{*}$ & -.43 & - & & \\
\hline \multicolumn{10}{|l|}{ Physical measure } \\
\hline 7 Walking speed & $-.53 *$ & .17 & -.25 & $-.40 *$ & -.14 & $-.49 *$ & .12 & - & \\
\hline \multicolumn{10}{|l|}{ Street-crossing decisions } \\
\hline 8 Collisions & $.59 *$ & $-.36^{*}$ & $.31 *$ & $.39 *$ & $.36^{*}$ & $.55^{*}$ & $-.27 *$ & $-.83 *$ & - \\
\hline
\end{tabular}


The correlation analyses revealed that collisions were significantly correlated with age and all functional abilities assessed. More precisely, a high proportion of collisions were associated with an old age, a low visual acuity score, a high motion-discrimination threshold, inhibition difficulties, flexibility errors, low attention scores, a decreased processing speed, and a slow walking speed. A forward step-wise regression analysis was then conducted to investigate the extent to which these factors account for age-related differences in the variance of collisions (Table 2).

Table 2. Summary of the forward step-wise regression analysis

\begin{tabular}{|c|c|c|c|c|c|}
\hline Steps & & $\mathrm{R}^{2}$ adjusted & $\mathrm{F}$ & $\mathrm{p}$ & beta \\
\hline 1 & $\begin{array}{l}\text { Walking speed } \\
\text { Inhibition } \\
\text { Flexibility } \\
\text { Attention } \\
\text { Processing speed } \\
\text { Visual acuity } \\
\text { Motion discrimination } \\
\text { Age }\end{array}$ & .79 & 218.35 & $<.0001$ & .89 \\
\hline 2 & $\begin{array}{l}\text { Walking speed } \\
\text { Flexibility } \\
\text { Inhibition } \\
\text { Attention } \\
\text { Processing Speed } \\
\text { Visual acuity } \\
\text { Motion discrimination } \\
\text { Age }\end{array}$ & .82 & $\begin{array}{c}228.81 \\
11.66\end{array}$ & $\begin{array}{c}<.00001 \\
<.001\end{array}$ & $\begin{array}{l}.85 \\
.19\end{array}$ \\
\hline 3 & $\begin{array}{l}\text { Walking speed } \\
\text { Flexibility } \\
\text { Visual acuity } \\
\text { Attention } \\
\text { Processing speed } \\
\text { Motion discrimination } \\
\text { Age }\end{array}$ & .83 & $\begin{array}{c}219.68 \\
8.67 \\
4.16\end{array}$ & $\begin{array}{c}<.00001 \\
<.01 \\
<.05\end{array}$ & $\begin{array}{r}.83 \\
.16 \\
-.12\end{array}$ \\
\hline
\end{tabular}

Note: dependent variable = collision; independent variables = age, cognitive, perceptual \& physical predictors; significant results indicated in bold $(p<.05)$

The forward step-wise analysis revealed that walking speed significantly predicted $79 \%$ of the variance in collisions. Cognitive flexibility added $3 \%$ of the variance explained by the model, and visual acuity $1 \%$. These three significant predictors accounted in total for $83 \%$ of the variance in collisions. As age was not a significant predictor on any of the steps, the results suggested that group differences in collisions were due to a significant decrease of walking speed, cognitive flexibility and visual acuity associated with aging.

\section{DISCUSSION AND CONCLUSIONS}

The present study showed that older pedestrians were more likely than younger adults to make street-crossing decisions that led to potential collisions with the approaching cars in a simulated pedestrian environment. Statistical analysis demonstrated that age-related physical, cognitive and perceptual limitations in elderly pedestrians predicted an increased likelihood of collisions. 
Limitations in physical abilities were shown to play the most important role in explaining the high percentage of collisions in older pedestrians, with slower walking speed being the strongest predictor of dangerous street-crossing decisions. This finding may be explained by the fact that older pedestrians can no longer compensate their selection of insufficient gaps to cross the street by walking faster. However, it is unlikely that the older participants of the present study had intentionally chosen to adopt this risky decision strategy. Instead, this finding may indicate an age-related difficulty in adapting decisions to changing abilities. Older pedestrians may have limited abilities or lack insight into their changing physical attributes and mobility, and may overestimate their walking speed. Consequently, they may tend to select too short gaps to cross the road and accommodate their slower walking speed. These results are in line with the recent study of Holland and Hill (2010) showing that the older pedestrians' awareness of their own walking speed is a causal factor in making dangerous street-crossing decisions.

The findings also slowed that poor cognitive flexibility was a significant predictor of the variance in collisions. Crossing the street can be considered a complex and goal-oriented task, therefore involving executive control processes. Cognitive flexibility, one component of the executive functions, is usually reported to decline with aging (Eppinger et al., 2007) and refers to the ability to shift lines of thought and actions in order to perceive, process and respond to situations in different ways (Eslinger \& Grattan, 1993). Our findings support the notion that, in a complex two-way road environment, older pedestrians seem to experience difficulties in efficiently switching their attention to the both sources of information and select among all the most relevant ones. This may be due to a reduced ability to adapt and shift their crossing strategy according to the road traffic oncoming information, such as speed of the approaching cars.

Finally, visual acuity appeared to be a significant predictor of the risk of being involved in a collision. Crossing the street is a highly visual-dependent task: pedestrians have to detect relevant information such as the ones relative to cars approaching in both ways in order to evaluate the available time to cross. The reduction of visual acuity with aging may contribute to misperceptions of the approaching car's speed and distance and thus explain the higher rate of poor crossing decisions in older pedestrians. These findings are in line with driving studies showing the role of age-related visual decline in crash risk (e.g. Shinar and Scheiber, 1991).

The results of the present study highlight a multi-dimensional explanation of the increase risk of being involved in a collision with aging. It includes a combination of physical, as well as cognitive and sensory performance decline with increasing age. Older people may have difficulties in efficiently compensating for their slower walking speed and visual acuity reduction when the complexity of the task exceeds their cognitive resources. While these findings have enhanced our understanding of behavioural and functional predictors of poor road crossing decisions, these responses were elicited in a laboratory setting using a simulated road-crossing environment and may be different to behaviours on the road. Further investigations of these associations in a real on-road task are warranted. Importantly, the implications of these findings relate to whether and how these difficulties can be counteracted by specific training. A possible effective approach could be a mixed physical-cognitive training to enhance the older pedestrians' motor abilities as well as executive control processes in complex perceptual-motor situations and help them become more aware of their own declining abilities. 


\section{REFERENCES}

Anstey KJ, Wood J, Lord S \& Walker JG. (2005). Cognitive, sensory and physical factor enabling driving safety in older adults. Clinical Psychology Review; 25, 45-65.

Crawford JR., Bryan J, Luszcz M A, Obonsawin M \& Stewart L (2000). The executive decline hypothesis of cognitive aging: Do executive deficits qualify as differential deficits and do they mediate age-related memory decline? Aging, Neuropsychology, and Cognition, 7, 9-31.

Eppinger, B., Kray, J., Mecklinger, A., \& John, O. (2007). Age differences in task switching and response monitoring: Evidence from ERPs. Biological Psychology, 75, 52-67.

Eslinger, P. J., \& Grattan, L. M. (1993). Frontal lobe and frontal-striatal substrates for different forms of human cognitive flexibility. Neuropsychologia, 31, 17-28.

Holland C, Hill R. (2010). Gender differences in factors predicting unsafe crossing decision in adult pedestrians across the lifespan: a simulation study. Accident Analysis and Prevention, 42:1097-1106.

Johnson CA and Keltner JL(1983). Incidence of visual field loss in 20000 eyes, and its relationship to driving performance. Archives of Ophtalmomlogy, 101, 371-375.

Ketchman CJ \& Stelman GE (2001). Age-related decline in motor control. In JE Birren, KW Schaie (Eds.) Handbook of the Psychology of Aging, 313-348. London: Academic Press.

Lobjois R, \& Cavallo V. (2009). The effects of aging on street-crossing behavior: from estimation to actual crossing. Accident Analysis and Prevention 41 (2), 259-267.

Lobjois R. \& Cavallo V. (2007). Age-related differences in street-crossing decisions: The effects of vehicle speed and time constraints on gap selection in an estimation task. Accident Analysis and Prevention, 39, 934-943.

Oxley JA, Fildes B, Ihsen E, Charlton J \& Day R. (1997). Differences in traffic judgments between young and old adult pedestrians. Accident Analysis and Prevention, 29, 839-847.

Oxley JA, Ihsen E, Fildes B N, Charlton J L. \& Day RH. (2005). Crossing roads safely: An experimental study of age differences in gap selection by pedestrians. Accident Analysis and Prevention, 37, 962-971.

Prull MW, Gabrieli JDE, \& Brunge SA (2000). Memory and Aging: a cognitive neuroscience perspective. In FIM Craik and TA Salthouse (Eds.), The Handbook of aging and cognition; 1-90. Mahwah NJ: Erlbaum.

Reitan, R. M., \& Wolfson, D. (1985). The Halstead-Reitan Neuropsycholgical Test Battery: Therapy and clinical interpretation. Tucson, AZ: Neuropsychological Press.

Rubin GS, West SK, Munoz B, Bandeen-Roche K, Zeger S, Schein O, Fried LP, \& the SEE Project team (1997). A comprehensive assessment of visual impairment in a population of older Americans. Investigative Ophthalmology and Visual Science, 38, 557-568.

Salthouse, TA. (1996). The processing-speed theory of adult age differences in recognition. Psychological Review, 103, 403-427.

Shinar D, \& Scheiber F (1991). Visual requirements for safety and mobility of older drivers. Human Factors, 33(5), 507-519.

Spreen O, and Strauss E. (1991). A compendium of neuropsychological test: administration, norms and commentary. New York: Oxford University Press. 
Wechsler D (2000). Manuel de l'échelle de l'intelligence pour adultes - 3eme Edition. Paris : Edition du centre de Psychologie appliquée.

Zimmerman P. \& Fimm B. (2009) Tests d'évaluation de l'attention. Freiburg: Psytest. 